\title{
Management for elevated serum Gamma Glutamyl Transpeptidase in primary
}

\author{
care settings
}

Sam Cheol Kim

Department of Family Practice and Community Medicine, Chosun University College of Medicine, Gwangju, Republic of Korea

\section{REVIEW}

Please cite this paper as: Kim SC. Management for elevated serum Gamma Glutamyl Transpeptidase in primary care settings. AMJ 2018;11(2):83-86.

https://doi.org/10.21767/AMJ.2017.3293

\section{Corresponding Author:}

Sam Cheol Kim, M.D.

Department of Family Practice and Community Medicine, Chosun University College of Medicine, 309 Pilmoondae-ro, Dong-gu, Gwangju 61452 Republic of Korea

Email: schkim@chosun.ac.kr

\section{ABSTRACT}

\section{Background}

One of the most frequently encountered problems in outpatient and health screening centres is the elevation of Gamma Glutamyl Transpeptidase(y-GTP) levels. The measurement of $y$-GTP is a very sensitive indicator for screening for the presence of hepatobiliary disease. Especially, when alkaline phosphatase is elevated, it is a useful test to distinguish the presence of cholestasis. The diseases for which y-GTP can be elevated include diabetes, pancreatic disease, fatty liver, primary and metastatic liver cancer, cirrhosis, neuromuscular disease, myocardial infarction, hyperthyroidism, chronic obstructive pulmonary disease, chronic renal failure, prostate cancer, rheumatoid arthritis, Obesity, drugs, etc. Since y-GTP has a relatively high sensitivity and low specificity, it is preferable to perform it with other blood tests rather than performing it independently. The measurement of y-GTP is used as a test to diagnose alcohol abuse.

\section{Aims}

What is the meaning of y-GTP when it is raised independently or in relation to other liver function tests and how is it interpreted? In this article, we will examine the meaning of $y$-GTP elevation and its clinical application in primary care settings.

\section{Key Words}

Measurement, y-GTP, primary care settings

\section{What this review adds:}

\section{What is known about this subject?}

Frequently, serum Gamma Glutamyl Transpeptidase(y-GTP) has been used to test liver function in general practice.

\section{What new information is offered in this review?}

This article shows check lists for history taking to identify an asymptomatic patient with abnormal liver tests, the meaning, structure, function of $y$-GTP and clinical interpretation of elevated y-GTP level.

3. What are the implications for research, policy, or practice?

In primary care settings, management strategy for elevated y-GTP cannot overemphasize too much. In fact, General practitioners tend to be pretty puzzled and confused. So this review will give you time to think this subject again.

\section{Introduction}

Liver function test (LFT) is a test that can easily be performed on the outpatient basis. Most of the patients with early-onset are first-time follow-up, for health screening and for follow-up during taking medication, regardless of their symptoms. Therefore, primary care physicians frequently encounter patients with abnormal liver function tests. It is very important to identify the cause, but it is not so easy to identify the cause in clinical practice. In particular, one of the most frequently encountered problems in primary care settings and health screening centers is the rise in Gamma Glutamyl Transpeptidase(yGTP) levels. In general, liver function tests include aspartate 
amino-transferase (AST), alanine aminotransferase (ALT), alkaline phosphatase (ALP), Gamma Glutamyl Transpeptidase ( $\mathrm{y}$-GTP), bilirubin, total protein and albumin. During the liver function test, AST, ALT and ALP reflect liver damage, albumin and prothrombin time (PT) show production capacity of liver, and bilirubin represents the excretion ability of liver. ${ }^{1}$ Therefore, in patients with mild liver function abnormalities, it is important to confirm the detailed history and to exclude common diseases causing abnormal liver function. In this case, follow-up observation may be necessary (Table 1 ).

Table 1: Check lists for history taking to identify an asymptomatic patient with abnormal liver tests

\author{
History of alcohol consumption \\ Family history of liver disease \\ Occupational exposure to toxins \\ Medications previous, current, herbal, and alternative \\ medicine \\ Evaluation for risk of viral hepatitis \\ Intravenous drug use \\ Sexual habitus \\ Tattoos \\ Non-sterile ear or body piercing or acupuncture \\ Blood or blood-product transfusions \\ Residence or travel to endemic areas \\ Metabolic factors: obesity, diabetes, and hyperlipidemia
}

In addition, since the liver function test is within the normal range and the patient cannot be said to have no chronic liver disease. If there are risk factors such as blood transfusion, alcohol drinking, and a family history with chronic viral hepatitis etc., in those cases, it may be required to confirm the absence of chronic liver disease in patients through additional tests. ${ }^{1-3}$

\section{Structure and function}

y-GTP is a glycoprotein enzyme that catalyzes the transfer of gamma-glutamyl of gamma-glutamyl peptides to other peptides and I-amino acids, and is involved in amino acid reabsorption, transport, and hydrolysis of glutathione. ${ }^{4}$ It is present in the canalicular membrane and biliary epithelial cells, part of the plasma membrane of hepatocytes. It also exists in other tissues such as the kidney, heart, pancreas, brain, and large intestine. The activity of y-GTP in the kidney is very active and is the most abundant in the brush border membrane in the tissue distribution. In the liver and pancreas, activity is low. ${ }^{5}$ However, it does not exist in the bone or placenta.
The biochemical, structural, and immunological knowledge of y-GTP has been extensively studied. However, the relationship between the test method and each disease and isoenzyme is still controversial. ${ }^{6}$ Therefore; the measurement of y-GTP is used as a very sensitive index for screening for the presence of liver dysfunction. It is used for the screening and diagnosis of intrahepatic and extrahepatic cholestasis or liver disease.

\section{Clinical interpretation}

y-GTP is present in hepatocyte and biliary epithelial cells and is a sensitive index for the presence of hepatocellular carcinoma, but its clinical usefulness is low because it is nonspecific. According to Table 2, y-GTP elevation can be observed in diseases such as pancreatic disease, myocardial infarction, renal failure, chronic obstructive pulmonary disease, diabetes, and alcohol abuse. y-GTP may be elevated when taking warfarin or phenytoin, carbarmazepine, barbiturate, or other anticonvulsants, and it should be excluded through history taking. ${ }^{7,8}$

\section{Table 2: Common causes of elevated gamma glutamyl} transpeptidase (y-GTP)

Hepatobiliary disease
Pancreatic disease
Alcoholism
Diabetes
Myocardial infarction
Chronic obstructive pulmonary disease
Renal failure
Drugs (carbamazepine, phenytoin, barbiturate, and
warfarin)

y-GTP is useful for the identification of alcohol consumption, suggesting alcoholic liver disease when the AST/ ALT ratio is 2 or more, together with the increase of $y$ GTP and aminotransferase. The sensitivity of $y$-GTP to alcohol abuse patients is known to range from 52 per cent to 94 per cent. However, y-GTP alone is limited in the diagnosis of alcohol abuse. Therefore, patients with y-GTP elevation should be considered together with other liver function items. ${ }^{1,9-12}$ In general, the normal range of y-GTP is somewhat different according to the literature, but broadly, it has a normal range of 2 65IU/L. According to one study, $y$-GTP increases as age increases in men, decreases after age 55 , and increases as age increases in women. ${ }^{13}$

The elevated levels of aminotransferase in liver function tests are different from those in which ALP or y-GTP is elevated alone. Elevated rates of AST or AST/ALT can be 
considered as initial damage findings by alcohol. In acute or chronic hepatitis, y-GTP is moderately elevated to less than 5 -fold the normal level. ${ }^{14}$ One study showed that $y$-GTP is not useful compared to aminotransferase in acute hepatitis but it is highly valuable in subacute or chronic hepatitis. In particular, y-GTP is elevated in recovery phase. In acute hepatitis, y-GTP is used to evaluate the progression of acute hepatitis and as a good indicator of progression to chronic hepatitis. ${ }^{15}$ Early diagnosis is needed because ALP and y-GTP increase several times compared with normal values after a few days after bile duct obstruction or intrahepatic cholestasis. It is known that y-GTP increases in about 90 per cent of patients with cholestasis. ${ }^{16}$ In some cases, it is helpful to measure y-GTP when ALP is elevated in patients without symptoms. The reason for this is that, in the case of liver disease, y-GTP is also increased proportionally as ALP increases. However, if ALP is elevated but normal y-GTP, then bone disease should be suspected. ${ }^{17}$ Therefore, y-GTP is a highly useful test that distinguishes whether the cause is liver disease or other diseases when ALP is elevated.

For another purpose, clinically, y-GTP is known to be the most affected by alcohol consumption. Therefore, the measurement of y-GTP is very helpful in diagnosing alcohol abuse. In chronic alcohol-intoxicated cases, all other liver function tests other than y-GTP are normal, so y-GTP is the most sensitive test for severe alcohol abuse. If you regularly take 60-80grams (more than 4 cups) of alcohol a day, you will have abnormal levels. Some surveys showed that even a low level such as 45grams (3 cups or more) makes it abnormal. ${ }^{18}$ However, when the amount is lowered, it may show normal values. In addition, when the AST: ALT ratio is at least 2:1 and y-GTP is twice the normal range, it strongly suggests alcohol abuse. Therefore, y-GTP can be a very useful indicator for follow-up management of alcohol. Generally, y-GTP is high in addicted drinkers. In particular, alcohol abusers who did not admit that they drank alcohol had a relatively high sensitivity (52-94 per cent) when they performed the test. ${ }^{19}$ The sensitivity was relatively high but the specificity was low so It is preferable to conduct the inspection in parallel. The elevation of y-GTP in alcohol abusers is thought to be mainly due to enzyme induction, although it may be due to liver cell damage or cholestasis. When the drinking history exceeds 10 years, the increase of $\mathrm{y}$-GTP is higher than the increase of ALP. While stopping drinking alcohol, y-GTP will be reduced in half after 26 days along with a relatively long half-life of alcohol. Thus, if y-GTP levels decrease after about 3 weeks, the increase in y-GTP may be attributed to alcohol consumption. In addition, if $y$ GTP is measured again after a certain period of time, it indicates that drinking is resumed. Therefore, it is used as an important index for observing well-being without alcohol consumption. However, the increase in y-GTP does not mean alcoholic hepatitis or fatty liver disease itself, but only chronic drinking. y-GTP can be used to detect alcohol abusers, but $1 / 3$ of them may show normal findings. Generally, it is known that alcohol-induced fatty liver does not occur in $<20 \mathrm{~g} /$ day in women and $<30 \mathrm{~g} /$ day in men. In addition, MCV, AST, AST/ ALT ratio, and mitochondrial AST are other methods to evaluate alcohol intake, but the clinical usefulness is low because of low sensitivity and specificity. $^{20}$

Even in neonates, y-GTP is useful for diagnosis of bile duct injury or cholestasis. Atresia may be suspected but not characteristic when the $\mathrm{y}$-GTP is elevated above 300IU/L in newborn infants. ${ }^{21}$

In 80 per cent of diabetic patients with fatty liver, liver function or $y$-GTP findings are abnormal. In the present study, y-GTP levels were significantly correlated with the degree of fatty liver, suggesting that y-GTP is a useful predictor of body fat mass. ${ }^{22}$ And y-GTP is an important predictor of obesity and fatty liver diagnosis because obesity increases and y-GTP level increases. It has been reported that $y$-GTP increases more than twice as the body mass index increases in men compared to women ${ }^{23}$ and serum triglycerides have an important effect on y-GTP. ${ }^{24}$ One study showed that y-GTP increased by 15 per cent in oral contraceptive use and decreased by 25 per cent in pregnant women and increased by 7 per cent after menopause. $^{25}$ If you are, you may be suspected of drug abuse. Taking drugs such as phenytoin, which induces microsomal enzymes, leads to y-GTP elevation. Aromatic drugs like Phenobarbital or phenytoin have characteristic findings of two-fold increase in the normal level of y-GTP. ${ }^{26}$ If $y$-GTP increases above 500IU/L with ALP, malignant tumors should be suspected. There is also a report that a member of a family has a high elevation of $y$-GTP. Isoenzymes showed high levels of GGT-2, autosomal dominant inheritance, and high levels of $y$-GTP did not necessarily indicate liver or biliary disease. ${ }^{27}$ There are reports that y-GTP is associated with stroke and early-onset morbidity at the middle age. ${ }^{28}$

\section{Conclusion}

As a conclusion, y-GTP is widely used to diagnose liver and biliary tract diseases. Especially, it shows a tendency to increase in the case of chronic alcoholic drinkers, which is very helpful in tracking control. However, in other diseases, y-GTP may be elevated and therefore differentiation is required. It is preferable to analyze the results in parallel 
with the results of other liver function tests rather than performing the tests alone because there is a disadvantage in that the specificity is low.

\section{References}

1. Ahn SY, Park SY. A Diagnostic Approach to Abnormal Liver Function Tests in Asymptomatic Patients. Korean J Med. 2012;82:134-42.

2. Pratt DS, Kaplan MM. Evaluation of abnormal liverenzyme results in asymptomatic patients. N Engl J Med. 2000;342:1266-71.

3. Green RM, Flamm S. AGA technical review on the evaluation of liver chemistry tests. Gastroenterology. 2002;123:1367-84.

4. Zuker SD, Gollan JL. Physiology of the liver. In: Haubrich WS, Schaffner F, Berk JE, eds. Bockus Gastroenterology. 5th ed. Philadelphia: WB Saunders; 1995.

5. Goldberg DM. Structural, functional, and clinical aspects of gamma-glutarryl- transferase. Crit Rev Clin Lab Sci. 1980;12:1-58.

6. Nemesanszky E, Lott JA. Gamma-glutamyltransferase and its isoenzymes:progress and problem. Clin Chem. 1985;31(6):797-803.

7. Vlahcevic ZR, Heuman DM. Diseases of the liver, gallbladder, and bile ducts. In Goldman L, Bennett JC, eds. Cecil textbook of medicine. 21st ed. Vol 1. Philadelphia: WB Saunders; 2000.

8. Pratt DS, Kaplan MM. Primary care: evaluation of abnormal liver-enzyme results in asymptomatic patients. N Engl J Med. 2000;342:1266-71.

9. Goldberg DM, Martin JV. Role of gamma-glutamyl transpeptidase activity in the diagnosis of hepatobiliary disease. Digestion. 1975;12:232-46.

10. Moussavian SN, Becker RC, Piepmeyer JL, et al. Serum gamma-glutamyl transpeptidase and chronic alcoholism: influence of alcohol ingestion and liver disease. Dig Dis Sci. 1985;30:211-4.

11. Orrego $\mathrm{H}$, Blake JE, Israel Y. Relationship between gamma-glutamyl transpeptidase and mean urinary alcohol levels in alcoholics while drinking and after alcohol withdrawal. Alcohol Clin Exp Res. 1985;9:10-3.

12. Rosalki SB, Tarlow D, Rau D. Plasma gamma-glutamyl transpeptidase elevation in patients receiving enzymeinducing drugs. Lancet. 1971;2:376-7.

13. Arnesen E, Huseby NE, Brenn T, et al. The Troms Heart Study: distribution of, and determinants for, gammaglutamyltransferase in a free-living population. Scan J Clin Lab Invest. 1986;46:63.

14. Kamath PS. Clinical approach to the patients with abnormal liver test results. Mayo Clin Proc. 1996;71:1089.
15. Whitfield JB, Pounder RE, Neale G, et al. Serum gammaglutamyl transpeptidase activity in liver disease. Gut. 1972;13:702-8.

16. Gopal DV, Rosen HR. Abnormal findings on liver function tests. Post Grad Med. 2000;107:100-14.

17. Moseley RH. Evaluation of abnormal liver function test. Med Clin North Am. 1996;80:887.

18. Whitehead TP, Clarke CA, Whitfield AG. Biochemical and hematological markers of alcohol intake. Lancet. 1978;1(8071):978-81.

19. Scheig R. Evaluation of test used to screen patients with liver disorders. Prim Care. 1996;23:551.

20. Lee KM. fatty liver. In: Lee HR, Kim SY, eds. Textbook of Family Medicine vol.2. Seoul: Hankuk medical publisher;2007:1234.

21. Sherlock S, Dooley J. Diseases of the liver and biliary system. 10th ed. Oxford: Blackwell Science; 1997.

22. Rosalki SB, Dooley JS. Liver function profiles and their interpretation. British J Hosp Med. 1994;51(4):181.

23. Choi K, Kim BI, Cho YK, et al. Diagnostic usefulness of serum r-glutamyl transferase(GGT) activity in fatty liver and relationship with other factors. Korean J Med. 1999;57(6):1006-13.

24. Schiele F, Guilmin AM, Detienne H, et al. Gammaglutamyltransferase activity in plasma: statistical distributions, individual variations, and reference intervals. Clin Chem. 1977;23:1023.

25. Fex G, Kristenson H, Trell E. Correlations of serum lipids and lipoproteins with gamma-glutamyltransferase and attitude to alcohol consumption. Ann Clin Biochem. 1982;19:345

26. Keeffe EB, Sunderland MC, Gabourel JD. Serum gammaglutamyl transpeptidase activity in patients receiving chronic phenytoin therapy. Dig Dis Sci. 1986;31:105661.

27. Guaitolini R. High serum gamma-glutamyltransferase concentrations in a family. $\mathrm{N}$ Engl J Med. 1994;330:1832-3.

28. Gill JS, Shipley MJ, Tsementzis SA, et al. Alcohol consumption-a risk factor for hemorrhagic and nonhemorrhagic stroke. Am J Med. 1991;90:489.

\section{PEER REVIEW}

Not commissioned. Externally peer reviewed.

\section{CONFLICTS OF INTEREST}

The author declares no competing interests.

\section{FUNDING}

None 